\title{
Mechanical, Electrical and Thermal Characteristics of HAF/ CR/LDPE Composites
}

\author{
F. Abd-El Salam ${ }^{1}$, L. A. Wahab ${ }^{2}$, M.H. Abd-EI Salam ${ }^{1}$ and \\ F. El- Sayed ${ }^{1}$
}

${ }^{1}$ Physics Department, Faculty of Education, Ain Shams University, Cairo, Egypt.

${ }^{2}$ National Center for Radiation Research and Technology, Cairo, Egypt.

The effect of low density polyethylene (LDPE) concentrations on the mechanical, electrical and thermal characteristics of $H A F / C R / L D P E$ composites has been studied. The elastic modulus values obtained from the stress - strain curves verify the requirements of the standard linear solid model. The first cycle hysteresis area $F A_{H}$ and the elastic recovery $\left(E_{R}\right)$ were found to be dependent on the (LDPE) content. The d.c. conductivity was found to decrease with increasing (LDPE) concentrations and showed a marked drop at the crystalline melting temperature of (LDPE) phase, $393 \mathrm{~K}$, followed by an increase above this temperature with average activation energy $0.79 \mathrm{eV}$. The crystalline melting temperature obtained from the conductivity - temperature relationship in agreement with that obtained by using differential scanning calorimetry (DSC). The temperature dependence of d.c. conductivity above 393 $K$ was in favour of the variable range hopping (VRH) model.

\section{Introduction:}

Polymer blending is one of the easiest and the most widely used techniques to prepare polymeric materials with desired properties. Blending a rubber with a plastic results in thermoplastic elastomers (TPEs). Although TPEs can be processed like a thermoplastic, yet they possess the properties of vulcanized elastomers. Thus TPEs bridge the gap between plastic and rubber industries. The mechanical properties of heterogeneous polymeric systems depend on the microstructure of the sample and polymer blends depend also on the size and shape of the domains, and the interactions between the components $[1,2]$. The static and dynamic elastic moduli of particulate composites, consisting of two phases one has isotropic- elastic properties and the other has linear viscoelastic properties, were studied by Theocaris et al [3]. Physical properties and processing behaviour for thermoplastic elastomer blends of polypropylenes (PP) and polybutadiene (BR) have been studied by Bhattachary et al [4]. The failure properties of HDPE and NBR blends has been discussed [5]. The effect of blend ratio, dynamic vulcanization and filler incorporation on the fracture behaviour was analyzed. Attempt has been made to correlate the 
fracture behaviour with the morphology of the sample and various theoretical models were introduced to support the experimental results. The stress-strain test is probably still the most widely used in the rubber industry for specification purposes and quality check on the compound. The electrical conduction process in conductive polymer composites is complicated and depends on a large number of parameters such as the filler (concentration, particle size, structure, filler matrix interaction) and processing techniques [6]. The incorporation of conductive fillers such as carbon black [7-9] enhanced the electrical properties of the polymer composites. The concentration and temperature dependence of the electrical conductivity of the non- crystallisable chloroprene Rubber CR (WRT) mixed with High abrasion furnace carbon black, (HAF) was investigated [10] and the temperature dependence of the electrical conductivity was discussed in view of the percolation theory. The system of carbon black / polyethylene mixtures shows a sharp resistivity increase with rise in temperature in the polymer melting region, i.e, there is a positive temperature coefficient (PTC) of resistivity, followed by a rapid resistivity decrease or negative temperature coefficient (NTC) [11]. The electrical transport in polyethylene -graphite composite materials was studied [12] and the analysis of the d.c. conductivity and complex permittivity in the frequency range from $10^{2}-10^{7} \mathrm{~Hz}$ was done. Differential scanning calorimetry (DSC) and x-ray studies were carried out [13] on a series of (NR - PE) thermoplastic elastomeric blends of various levels of interaction. The chemical structure of rubber and its composition in the blend do not affect significantly the $\left(T_{m}\right)$ of PE. The addition of rubber causes a tendency for the interplanar distance (R) to decrease in all the systems, which points to an appreciable migration of rubber into the interchain space of (PE). The aim of this work is to investigate the effect of the blend ratio of LDPE/CR(WRT) blend on the mechanical, electrical and thermal properties of $\mathrm{HAF} / \mathrm{CR} / \mathrm{LDPE}$ composite.

\section{Experimental Work:}

\subsection{Materials}

The elastomer used in the present study was the non- crystallisable chloroprene Rubber CR (WRT), having specific gravity of 1.23 at $296 \mathrm{~K}$, mixed with different ratios of low density polyethylene (LDPE), of specific gravity $0.91-0.94$, crystalline melting point, $\boldsymbol{T}_{\boldsymbol{m}}=393 \mathrm{~K}$ and volume resistivity $10^{16} \Omega . c m$. High abrasion furnace carbon black, N-330 (HAF): with granulated powder of $40 \mathrm{~nm}$ particle size and specific gravity $1.78-1.82$ was used as conductive filler. The choice of CR (WRT) is due to its excellent long- term stability, at least in its vulcanized form; and hence its promise for practical industrial applications. All samples were prepared according to the recipe presented in table (1). The samples under test were designated as xLy, where $\mathrm{x}$ and $y$ stand for the concentrations of HAF- black and LDPE in (phr), 
respectively.

Table (1): Recipe for HAF/ CR composites containing different concentrations of LDPE.

\begin{tabular}{|c|c|c|c|c|c|c|}
\hline Ingredients & OLO & 0L10 & $40 \mathrm{L10}$ & $40 \mathrm{L30}$ & $40 \mathrm{L50}$ & $40 L 70$ \\
\hline CR (WRT) & 100 & 90 & 90 & 70 & 50 & 30 \\
\hline LDPE & 0 & 10 & 10 & 30 & 50 & 70 \\
\hline Stearic acid & 1 & 1 & 1 & 1 & 1 & 1 \\
\hline Zinc oxide & 5 & 5 & 5 & 5 & 5 & 5 \\
\hline Processing oil & 10 & 10 & 10 & 10 & 10 & 10 \\
\hline HAF- black & 0 & 0 & 40 & 40 & 40 & 40 \\
\hline MBTS $^{a}$ & 1 & 1 & 1 & 1 & 1 & 1 \\
\hline TMTD $^{b}$ & 1 & 1 & 1 & 1 & 1 & 1 \\
\hline Sulphur & 1.5 & 1.5 & 1.5 & 1.5 & 1.5 & 1.5 \\
\hline
\end{tabular}

The rubber composites and blends were prepared on a two rolls mill of $300 \mathrm{~mm}$ length, $170 \mathrm{~mm}$ diameter with speed of slow roll $18 \mathrm{rev} . / \mathrm{min}$. and gear ratio 1.4. After mixing, the rubber compositions were left for about 24 hours and then molded in an electrically heated hydraulic press at $143 \pm 2{ }^{\circ} \mathrm{C}$ under a pressure of $4 \mathrm{MPa}$ and the time of vulcanization was $20 \mathrm{~min}$. The samples were prepared by using the facilities of the Transport and Engineering (Rubber Manufacturing) Company, Alexandria, Egypt.

\subsection{Measurements:}

Dumbell shaped samples were cut from the vulcanized sheets by a fine edge steel die with a constant width of $4 \mathrm{~mm}$. The thickness of the test sample was determined with a dial gauge. The apparatus used for stress-strain measurements was described elsewhere [14]. The samples were stretched at a constant speed $10 \mathrm{~mm} / \mathrm{min}$. adjusted by means of dc motor. The applied force was recorded continuously during the extension of the sample up to rupture.

The dc conductivity apparatus was described previously [15]. The dc conductivity was measured from room temperature up to about $420 \mathrm{~K}$. The DSC measurements were carried out from room temperature up to $430 \mathrm{~K}$ by using Shimadzu (50) differential scanning analyzer with a scanning speed of $5^{\circ} \mathrm{C} / \mathrm{min}$. in the microanalysis unit at the national center for radiation research and technology, Cairo, Egypt.

\section{Results and Discussion:}

\subsection{Stress-strain Measurements:}


The stress-strain curves of (LDPE/ CR) rubber blends with different blend ratio either unloaded or loaded with $40 \mathrm{phr}$ of HAF-carbon black were obtained and the experimental results (points) are shown in Fig (1). The solid lines show the theoretical data obtained by applying the standard linear solid model as described later.
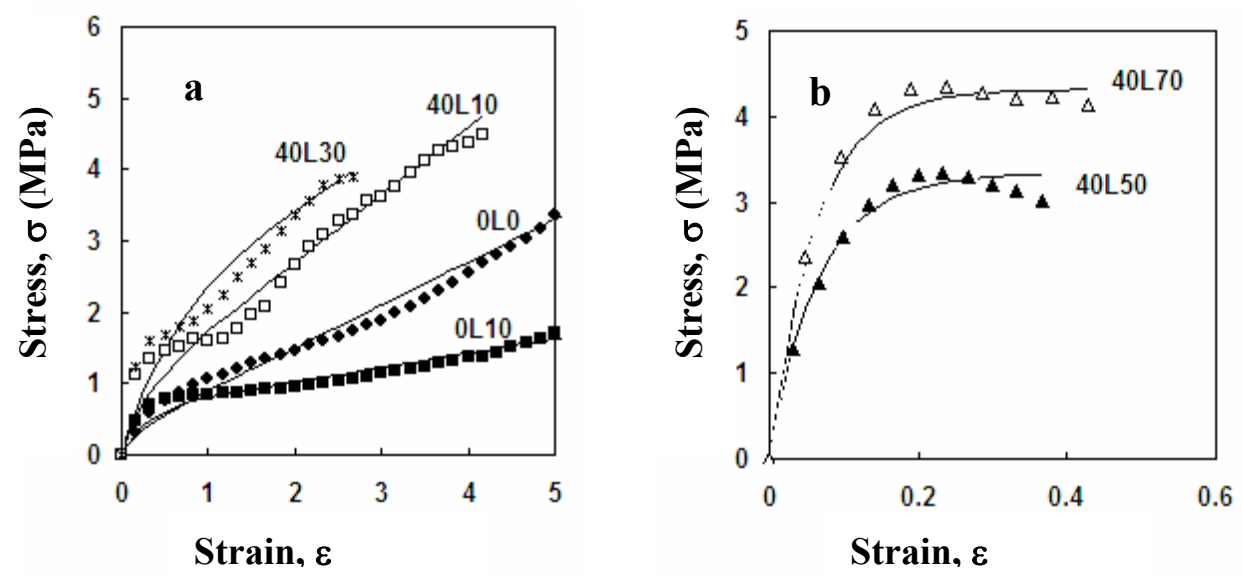

Fig.1. Stress-strain curves for (LDPE/ CR) rubber blends with different blend ratio loaded with HAF-carbon black. The points represent the experimental data and the solid lines are due to the standard linear solid model (eq. (2)).

From Fig.(1) it is clear that the stiffness, as governed by the slope of the initial linear part or the modulus of elasticity $E$, increases with increasing the concentration of LDPE which acts as a filler. Accordingly, the elongation at break decreases (see table 2). The stress-strain behaviour of the samples 40L50 \& 40L70, considered as plastic rich blends, showed very high initial modulus with yielding and necking. The drastic increase in the elastic modulus may be due to the higher proportion of plastic phase and the transition of the continuous rubber phase into a fine crosslinked rubber particles dispersed in a plastic matrix. On increasing the proportion of PE from 50 to 70 phr Fig. (1b) shows that the matrix can withstand higher stress and consequently an increased extent of deformation before failure, i.e. increased tensile strength and elongation at break. This may be ascribed to the decrease of the diffusional mobility of the crosslinked rubber chains.

It is well known that a stress-strain curve of a polymer is often mathematically described by models indicating the visco-elastic behaviour of the polymer [16]. In the standard linear solid model, the visco-elastic behaviour of a polymer is described by a spring (with elastic modulus $E$ ) and a dashpot (with damping constant or viscosity $\boldsymbol{\eta}$ ) in series with each other, and both are in parallel with a long spring (with elastic modulus $E^{\prime}$ ). For this model, which is applicable to the present work, the governing constitutive relation is[17]: 


$$
\frac{d \sigma}{d t}+\frac{E}{\eta} \sigma=\frac{E E^{\prime}}{\eta} \varepsilon+\left(E+E^{\prime}\right) \frac{d \varepsilon}{d t}
$$

Under a constant strain rate, $(\gamma=d \varepsilon / d t)$, the solution of equation (1) can be written as:

$$
\sigma=\eta \gamma\left[1-e^{-\left(\frac{E \varepsilon}{\eta \gamma}\right)}\right]+E^{\prime} \varepsilon
$$

This equation contains three fitting parameters, $E, E^{\prime}$ and $\eta$. Appling eq. (2), the solid lines in Fig. (1) are obtained and the fitting parameters are calculated and listed in Table (2). It is clear that, there is an agreement between the theoretical and the experimental data of the stress - strain curves. The fitting parameters $E, \eta$ increase with increasing LDPE concentration, meanwhile the values of $E^{\prime}$ decrease. From Table (2), it is also clear that the calculated values of the spring elastic modulus $E$ of the theoretical model are very close to those obtained experimentally from the slopes of the initial linear portions of the stress - strain curves. This indicates that the series spring in this model $E$ plays the dominant role in the early stage of the stress - strain curves,

i.e. before reaching the yield point, while the parallel spring and therefore the modulus $E^{\prime}$ plays the dominant role after the yield point of the stress - strain

Table (2): The fitting parameters, as calculated by applying the standard linear solid model for the investigated composites, and the experimental values of the elastic modulus $\mathrm{E}$.

\begin{tabular}{ccccc}
\hline Sample & $\begin{array}{c}E \\
(\mathbf{M P a})\end{array}$ & $\begin{array}{c}\eta \\
(\text { GPa.sec) }\end{array}$ & $\begin{array}{c}E^{\prime} \\
(\mathbf{M P a})\end{array}$ & $\begin{array}{c}E_{\text {exp erimental }} \\
(\mathbf{M P a})\end{array}$ \\
\hline $\mathbf{0 L 0}$ & 1.26 & 0.90 & 0.60 & 1.52 \\
0L10 & 1.52 & 1.89 & 0.30 & 1.52 \\
40L10 & 2.77 & 2.40 & 0.90 & 2.77 \\
40L30 & 3.26 & 6.00 & 0.75 & 3.27 \\
40L50 & 52 & 9.90 & 0.10 & 25.52 \\
40L70 & 70 & 12.90 & 0.05 & 28.25 \\
\hline
\end{tabular}

curves. From Table (2), it is also noticed that $E^{\prime} \cong E / 3$, for samples containing LDPE concentrations from 0 to $30 \mathrm{phr}$. Therefore, the fitting parameter $E$ represents the Young's modulus while E' can be considered as the shear or rubbery modulus [18]. In contrast, for the samples with higher content of LDPE 
(50 and $70 \mathrm{phr}$ ) the relation $E^{\prime}=E / 3$ does not hold. In fact, PE is introduced into the matrix as a filler, so it raises Young's modulus (E) as mentioned before and impedes the formation of physical cross-linking by carbon black, which reduces the values of the rubbery modulus. Besides, for blends not containing carbon black, the decrease in $E^{\prime}$ might be due to the existence of grain boundaries between the two phases. Furthermore, the values of $\eta$ as calculated from this fitting correspond to the viscosity of the composites.

\subsection{Cyclic Stress - Strain Curves (Hysteresis)}

Under cyclic loading, rubber dissipates energy due to hysteresis effects [19], which is caused by a breakdown of crosslinks and a progressive detachment of rubber molecules from the surfaces of reinforcing fillers. Figure (2) shows the first and second cycles of the viscoelastic cyclic stress-strain of the tested samples. The elastic recovery $E_{R}$ and the first cycle hysteresis area $F A_{H}$ has been calculated for different concentrations of LDPE and listed in table (3).

It is clear that $E_{R}$ decreases with increasing the proportion of LDPE, meanwhile, $F A_{H}$ attains its maximum value at $30 \mathrm{phr}$ of LDPE. For carbon free samples, $0 \mathrm{~L} 0 \& 0 \mathrm{~L} 10$, the addition of PE increases $F A_{H}$ while $E_{R}$ decreases. In fact, $\mathrm{PE}$ in this matrix is not crosslinked. Accordingly, PE exists in lamellar state introducing internal stresses which retard the elastic deformation and increase the energy loss. Consequently, $F A_{H}$ is expected to increase and $E_{R}$ to decrease. It is also noticed that, at a definite PE content [samples, 0L10 \& 40L10], the hysteresis is smaller for the carbon free sample compared to that filled with HAF-black. The presence of particulate filler such as carbon black, leads to decreased segmental mobility and hence increased viscosity and internal friction between rubber chains leading to increased hysteresis[20]. This is the result of rearrangement of the molecular structure under the applied load and subsequent sliding of chains, past each other. Increasing the proportion of $\mathrm{PE}$ above 30phr, in plastic rich blends, decreases $F A_{H}$. The suppression of coalescence resulting from the decreased diffusional mobility of the dispersed crosslinked rubber chains is believed to be the main reason for the decrease in the hysteresis area $F A_{H}$ with increasing LDPE content.

The second cycle hysteresis is smaller than that of the first cycle one. However, in the third cycle the amount of hysteresis is almost the same as the precedent one. This behaviour, known as stress softening, is due to the modification and reformation of rubber network structures which leads to weak hysteresis variations. The main features contributing to the stress-softening behaviour involve chemical effects, microstructural damage, multi-chain damage, and micro-void formation [21].
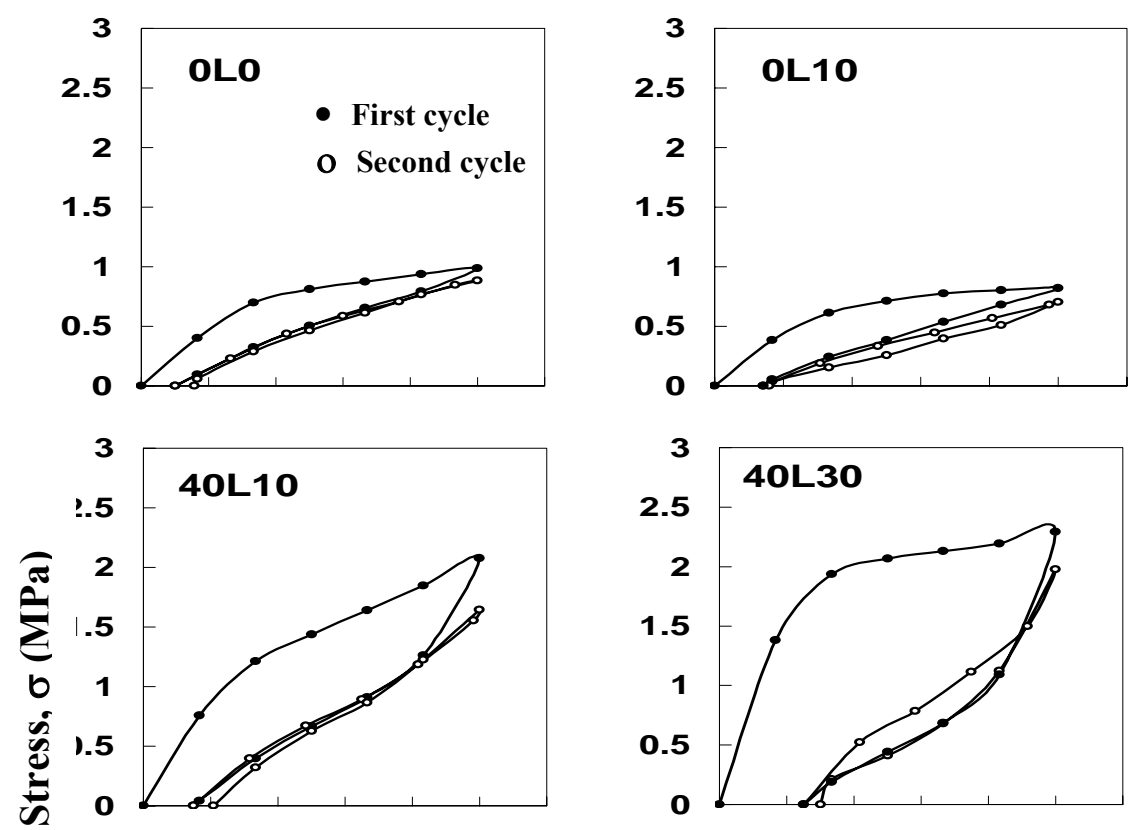
Table (3): The values of elastic recovery $E_{R}$ and the first cycle hysteresis area $\mathrm{FA}_{\mathrm{H}}$.

\begin{tabular}{ccc}
\hline Sample & $E_{R}$ & $F A_{H}$ \\
\hline 0L0 & 0.90 & 1.65 \\
0L10 & 0.87 & 2.02
\end{tabular}




$\begin{array}{lll}\text { 40L10 } & 0.85 & 2.69 \\ \text { 40L30 } & 0.75 & 3.72 \\ \text { 40L50 } & 0.73 & 1.45 \\ \text { 40L70 } & 0.50 & 0.59\end{array}$

\subsection{Electrical Conductivity:}

Fig.3 depicts the temperature dependence of the dc electrical conductivity of (LDPE/ CR) rubber blends with different blend ratios loaded with HAF-carbon black. It is clear that the dc conductivity decreases with increasing the proportion of LDPE. On increasing temperature the conductivity decreases,i.e.,a negative temperature coefficient of conductivity (NTCC) is noticed up to $(\cong 393 \mathrm{~K})$. Above this temperature a usual thermally activated behaviour similar to the case of intrinsic semiconductors is observed. This condition is probably associated with the melting of the crystalline phase of LDPE at $\left(\mathrm{T}_{\mathrm{m}}\right)$ and the consequent increase in the free volume which results in the observed decrease of the electrical conductivity. At higher temperatures $(\mathrm{T}>393 \mathrm{~K})$ in fig. 3 a thermally activated part appears with average activation energy of $0.79 \mathrm{eV}$. This thermally activated region is thought to be related to

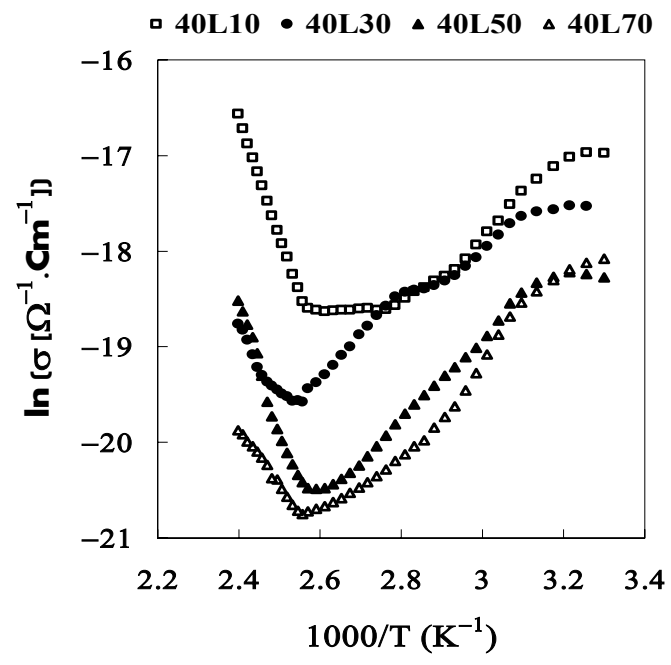

Fig. (3): The temperature dependence of dc electrical conductivity for (LDPE/ CR) rubber blends with different blend ratios loaded with HAF-carbon black.

the melting of the (PE) crystallites and the redistribution of HAF- black particles.

The data collected from the dc conductivity measurements were consistent with the observed DSC results. Fig.4 depicts differential scanning calorimetry thermograms for some of the studied composites. The values of $T_{m}$ and the heat of fusion $\Delta H$, which can be considered as a measure of the 
fractional crystallinity [22] of the tested blends, are given in Table (4).

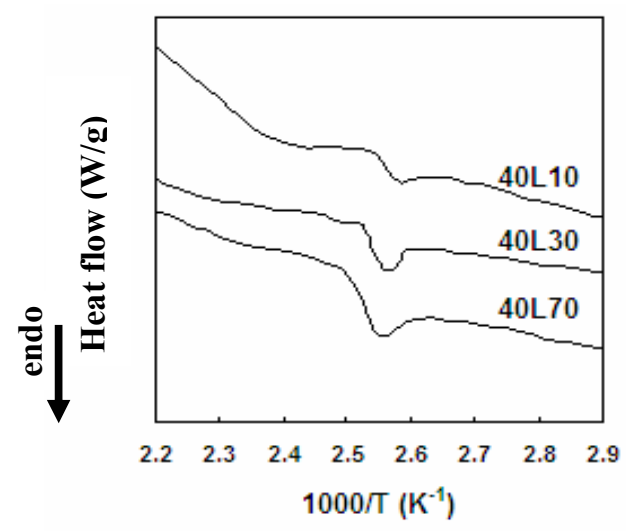

Fig. (4): Differential scanning calorimetry thermographs for some of the studied composites.

It is clear that the values of $\Delta H$ increase with increasing the concentration of $\mathrm{PE}$, as expected for the crystalline phase. Comparison between the DSC thermograms and the temperature dependence of conductivity $\sigma(\mathrm{T})$ [see figs.3,4] shows a clear coincidence between the observed minima for both $\sigma(\mathrm{T})$ and DSC thermograms. Thus, it may be concluded that the temperature dependence of conductivity can be used to determine $\left(\mathrm{T}_{\mathrm{m}}\right)$ for the crystalline phase.

To further pursue analysis of the experimental results, a trial was made to determine the dominant conduction mechanism. The variable range hopping (VRH) model proposed by Mott [23] has been employed. According to (VRH) model, the temperature dependence of conductivity follows the formula[24].

Table (4): The derived values of $\mathrm{T}_{\mathrm{m}}$ and $\Delta H$ from the DSC thermographs of fig.4.

\begin{tabular}{ccc}
\hline Sample & $\mathbf{T}_{\mathbf{m}}(\mathbf{K})$ & $\Delta \mathbf{H}(\mathbf{J} / \mathbf{g})$ \\
\hline $40 \mathrm{~L} 10$ & 391 & 1.38 \\
$40 \mathrm{~L} 30$ & 390.6 & 3.26 \\
$40 \mathrm{~L} 50$ & 391 & 4.90 \\
$40 \mathrm{~L} 70$ & 390 & 5.80 \\
\hline \multicolumn{3}{c}{$\sigma T^{1 / 2}=\sigma_{\circ} \exp \left(-T_{\circ} / T\right)^{\gamma}$}
\end{tabular}

where $\sigma_{\circ}$ is the limiting value of conductivity at infinite temperature, $T_{\circ}$ is the characteristic temperature which determines the thermally activated hopping among localized states at different energies and is considered as a measure of disorder [25] and the exponent $\gamma$ is related to the sample thickness $d$, via the 
equation $\gamma=1 /(1+d)$. In this study the average thickness $d$ of the samples is $\approx 2 \mathrm{~mm}$, i.e. $\gamma$ is equal to $(1 / 3)$. Fig. 5 correlates $\ln \left(\sigma T^{1 / 2}\right)$ with $T^{-1 / 3}$.

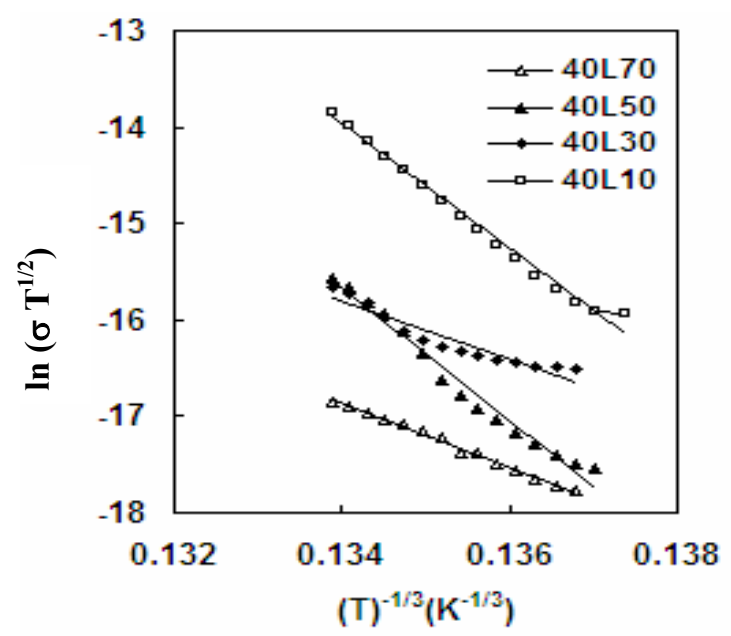

Fig. (5): Variation of $\ln \left(\sigma T^{1 / 2}\right)$ with $T^{1 / 3}$ for the tested samples in the temperature range from 393 to $413 \mathrm{~K}$.

The linear curves obtained in the investigated temperature range, $383-413 \mathrm{~K}$, indicate that the (VRH) model is applicable [26] as the predominant mechanism of conduction in the present work. The values $T_{\circ}$ for different frequencies, derived from the linear fits are listed in Table (5). It is clear that, the high values of $T_{\circ}$ indicate a low conductivity for the system and that it decreases with increasing the concentration of PE. As mentioned before, $T$ 。 reflects the structural disorder of the matrix. In general, increasing PE proportion increases the crystalline phase of the composite as a whole which in turn increases the degree of ordering within the matrix. The peculiar increase of $T_{\text {。 }}$ for the sample 40L50 may be attributed to the transition of the continuous rubber phase to fine crosslinked rubber particles dispersed in the plastic matrix.

Table (5): Derived values of the characteristic temperature $T_{\circ}$ according to the (VRH) Model.

\begin{tabular}{|c|c|c|c|c|}
\hline Sample & $40 \mathrm{~L} 10$ & $40 \mathrm{~L} 30$ & $40 \mathrm{~L} 50$ & $40 \mathrm{~L} 70$ \\
\hline $\mathbf{T}_{\mathbf{0}}(\mathbf{K})$ & $2.67 \times 10^{8}$ & $2.69 \times 10^{7}$ & $3.22 \times 10^{8}$ & $3.67 \times 10^{7}$ \\
\hline
\end{tabular}

\section{Conclusion:}


The stress- strain measurements of HAF/CR/LDPE composite indicate that $\mathrm{PE}$ is introduced into the matrix as a filler, so it raises Young's modulus (E) and impedes the formation of physical cross-linking by carbon black, which reduces the values of rubbery modulus. The transition of the continuous rubber phase into fine crosslinked rubber particles dispersed in the plastic matrix causes the drastic increase in the elastic modulus of the blend with higher proportion of LDPE. The elastic modulus values obtained from the stress strain curves are close to those obtained from the standard linear solid model. The elastic recovery $E_{R}$ was found to decrease with increasing the proportion of LDPE, and $F A_{H}$ showed a maximum value at $30 \mathrm{phr}$ of LDPE. The incorporation of LDPE decreases the electrical conductivity. The temperature dependence of conductivity showed a negative temperature coefficient of conductivity (NTCC) up to $(\cong 393 \mathrm{~K})$, which was attributed to the melting of the crystalline phase of LDPE $\left(\mathrm{T}_{\mathrm{m}}\right)$ and the consequent increase in the free volume which results in the observed decrease of the electrical conductivity. At higher temperatures $(\mathrm{T}>393 \mathrm{~K})$ a thermally activated part appeared with average activation energy of $0.79 \mathrm{eV}$. The values of the melting temperature of the crystalline phase $\left(\mathrm{T}_{\mathrm{m}}\right)$, deduced from the temperature dependence of conductivity are comparable with those determined from DSC thermograms. The linear curves obtained in the investigated temperature range,383-413 K, indicate that the (VRH) model is applicable as the predominant mechanism of conduction in the present work.

\section{References:}

1. E. Fekete, B. Pukanszky, and Z. Perdy; Die Angew. Makromol. Chem., 199, 87 (1992).

2. G.Qipeng, Q. Lingwei, D.Mengxian, and F. Zhiliu; Eur.Polym.J., 28, 1045 (1992).

3. P.S. Theocaris and E.S. Sideridis; J. Appl Polym. Sci., 29, 2997 (1984).

4. B.Bhattachary, Baby Kuriakose, B.R. Gupta, and S.K. De; International Rubber Conference, Moscow, (1984).

5. N. R. George, K. T. Neelakantan and S. T. Varughese, J. Applied Polymer Science, 100, 2912 (2006).

6. F. El-Tantawy, European Polymer Journal 38, 567 (2002).

7. Y. Bin, C. Xu, Y. Agari and M. Matsuo, Colloid Polym Sci, 277 (5), 452 (1999).

8. Y. Bin, C. Xu, D. Zhu and M. Matsuo, Carbon, 40 (2), 195-9 (2002).

9. M. Hindermann-Bischoff and F. Ehrburger-Dolle, Carbon, 39 (3), 375-82 (2001).

10. H. Ali and A. Abo-Hashem, J. Materials Processing Technology, 68, 163- 67 (1997). 
11. J. Meyer; Polym. Eng. Sci., 14, 706 (1974).

12. A.Ezquerra, M. Kulescza, and F. J. Balta-Calleja, Synthetic Metals, 41, 915 (1991).

13. N.R. Choudhury, T.K. Chaki, A. Dutta, and A.K. Bhowmick; Polymer, 30, 2047 (1989).

14. M.H.Abd El-Salam, Ph.D. Thesis, Fac- Sci. Cairo univ. Cairo, Egypt, 41 (1997).

15. E.M. Abdel Bary, M. Amin and H. H. Hassan, J. Polym Sci, 15, 147 (1977).

16. Tager, "Physical Chemistry of polymers", Mir Pub., 2nd Ed., Moscow, (1978).

17. L.E. Nielsen and R.F. Landel, "Mechanical Properties of Polymers and Composites", 2nd ed., Revised and Expanded, (1994).

18. C.E. Locke and D.R. Paul ; Polym. Eng. Sci. , 3, 308 (1973).

19. G. Kraus, Adv. Polym. Sci., 8, 155 (1971).

20. W. Ren, Colloid Polym. Sci., 270, 990 (1992).

21. J. A. Harwood and A. R. Payne, J. App. Polym. Sci., 10, (1966).

22. M.T. Chanliau-Blanot, M.Nordin, J. B. Donnet, E. Papirer, G. Roche, P.Laurenson, and G.Rossignol; J. Mater. Sci., 24, 641 (1989).

23. N.F. Mott and E.A. Davis, "Electronic conduction in non-crystalline materials", Oxford: Clarenton Press; 157-60 (1979).

24. N.F. Mott, "Conduction in non-crystalline materials", Oxford: Clarenton Press; (1987).

25. P. Mandal, A. Neumann, A.G.M. Jansen and P. Wyder, Phys Rev B 55, 452-6 (1997).

26. G.C. Psarras, Composites: Part A 37, 1545-1553 (2006). 